\title{
High Incidence of Device-Related and Lead- Related Complications in the Dual-Chamber Implantable Cardioverter Defibrillator Compared With the Single-Chamber Version
}

\author{
Tamana Takahashi, MD; Anil K. Bhandari, MD; Masato Watanuki, MD; \\ David S. Cannom, MD; Harumizu Sakurada, MD*; Masayasu Hiraoka, MD*
}

\begin{abstract}
Recently, the routine use of dual-chamber implantable cardioverter defibrillators (DC-ICD) has been advocated over the single-chamber version (SC-ICD), but there are few reports of the frequency of complications between the 2 types of ICDs. Between July 1997 and April 1999, 178 consecutive patients underwent implantation of either a transvenous SC-ICDs $(n=104)$ or a DC-ICDs $(n=74)$. Twelve $(16 \%)$ of the 74 patients with a DC-ICD had a total of 16 major complications compared with $6(6 \%)$ of the 104 patients with a SC-ICD ( $=0.01)$. The 16 DC-ICD complications included atrial lead dislodgment (4), ventricular lead malfunction (4), and pocket infection/hematoma (3), and the 6 SC-ICD complications included ventricular lead dislodgment (2) and pocket hematoma (3). Patients with a DC-ICD had less left ventricular function (29\% vs 35\%, p=0.035) and a higher prevalence of non-ischemic cardiomyopathies ( $48 \%$ vs $28 \%, \mathrm{p}=0.0076)$. In conclusion, the DC-ICD may have a higher frequency of device- and lead-related major complications. (Circ J 2002; 66: 746-750)
\end{abstract}

Key Words: Complications; Dual-chamber ICD; Single-chamber ICD

W

ith rapid progress in the design and technological aspects of the implantable cardioverter defibrillator (ICD), its use has increased dramatically over the past decade!-3 At the same time, complications associated with the pectoral defibrillator and its transvenous leads have been recognized more frequently than before ${ }^{4-6}$ Because it uses an atrial catheter, the dual chamber ICD (DC-ICD) can be used for DDD or DDD-R pacing with improved discrimination between ventricular and nonventricular arrhythmias, 8 although complications have been frequently associated with this complex lead system.

In order to assess the risk of this new treatment system, we studied the results of short- and long-term follow-up of patients with DC-ICD and single-chamber ICD (SC-ICD) in terms of the frequencies and types of major complications requiring surgical correction.

\section{Methods}

Between July 1997 and April 1999, 178 consecutive patients underwent implantation of either a DC- $(n=74)$ or SC-ICD $(n=104)$ under general anesthesia. The DC-ICD was selected for patients with the complications of sick sinus syndrome, atrio-ventricular conduction disturbance or supraventricular tachycardia, and by hemodynamic factors after the shock delivery. Cases of ICD replacement were

(Received January 17, 2002; revised manuscript received April 30, 2002; accepted May 2, 2002)

Department of Cardiology, Good Samaritan Hospital, Los Angeles, California, USA and *Department of Cardiovascular Disease, Medical Research Institute, Tokyo Medical and Dental University, Tokyo, Japan Mailing address: Tamana Takahashi, MD, Department of Cardiology, Good Samaritan Hospital, 1225 Wilshire Blvd., Los Angeles, CA 90017, USA excluded from this study. We retrospectively studied the following variables when reviewing the charts of all patients: age, sex, left ventricular ejection fraction (LVEF), ICD generator model, lead model, cardiac disease, device indication, presence of adverse events and complications during the follow-up period. Each patient received a transvenous lead system (Guidant; Endotak DSP, Endotak Endurance, Medtronic; CapsureSP, Sprint Ventritex: SPL) and ICD pulse generator (Guidant; Ventak Mini, Ventak AV, Medtronic; Micro Juel, Gem, GemDR, Ventritex; Angstrom, Contour, Profile) in any one of these combinations. After the leads were correctly positioned, the efficacy of pacing, sensing, and defibrillation was assessed. One week after discharge from hospital, the patients visited the outpatient office and then returned every 3 months for regular check-ups or in the event of any adverse event during the follow-up period. At each visit, an interim history was obtained in regard to the presence or absence of syncopal episodes, palpitations and device discharge. Device evaluation included capacitor reformation and device interrogation to determine whether or not episodes of tachycardia were detected or the shocks were delivered. Measurements of lead impedance, pacing, and sensing thresholds were also done. For this analysis, major complications were defined as those requiring surgical correction. The time to follow-up was the period up to the most recent follow-up visit or the patient's death.

\section{Statistical Methods}

Continuous variables are presented as mean \pm standard deviation (SD), unless otherwise indicated. For discrete variables the continuity adjusted chi-square test was used. 
Table 1 Baseline Clinical Characteristics

\begin{tabular}{lccc}
\hline \hline & Total & Dual-chamber ICD & Single-chamber ICD \\
\hline No. of patients & 178 & 74 & 104 \\
Men/women & $144 / 34$ & $58 / 16$ & $86 / 18$ \\
Age (years) & 64 & 66 & 62 \\
Cardiac disease & & 38 & 70 \\
$\quad$ CAD & 108 & 29 & 18 \\
$\quad$ Cardiomyopathy & 37 & 1 & 7 \\
RVD & 8 & 2 & 8 \\
Long QT & 3 & 4 & $35 \pm 16$ \\
Other & 12 & $29 \pm 12$ & 29 \\
Ejection fraction (\%) & $33 \pm 15$ & 14 & $24 / 13$ \\
Induction & 43 & $19 / 10$ & 38 \\
$\quad$ Aborted SCD & $43 / 23$ & 31 & $12 \pm 6$ \\
Syncopelnear syncope & 69 & $9 \pm 6$ & \\
None & $11 \pm 6$ & & \\
Months of follow up & & & \\
\hline
\end{tabular}

ICD, implantable cardioverter defibrillator; $C A D$, coronary artery disease; $R V D$, right ventricular dysplasia; SCD, sudden cardiac death.

Table 2 Complications With the 2 Type of ICD

\begin{tabular}{lcc}
\hline \hline & Dual-chamber ICD & Single-chamber ICD \\
\hline Generator malfunction & $2(2.7 \%)$ & 0 \\
Pocket hematoma & $1(1.4 \%)$ & $3(2.9 \%)$ \\
Infection & $3(4.1 \%)$ & 0 \\
Pneumothorax & 0 & $1(1.0 \%)$ \\
Atrial lead dislodgement & $4(5.4 \%)$ & - \\
Atrial lead fracture & $1(1.4 \%)$ & - \\
Ventricular lead malfunction & $1(1.4 \%)$ & $2(2.0 \%)$ \\
Ventricular lead dislodgement & 0 & 0 \\
Ventricular lead fracture & $3(4.1 \%)$ & 0 \\
Right ventricular perforation & $1(1.4 \%)$ & \\
\hline
\end{tabular}

ICD, implantable cardioverter defibrillator.

Table 3 Lead Implantation: Complications, Presentation and Time From Implantation

\begin{tabular}{llc}
\hline \hline Complication (device model) & Presentation & Time from implant \\
\hline SC-ICD: & & \\
V-lead dislodgment $(1762)$ & Inhibiting pacing via ICD V-lead & 2 days \\
V-lead dislodgment $(7223 C X)$ & Inappropriate shocks & 15 months \\
DC-ICD: & Inappropriate shocks & \\
V-lead sensing malfunction (1831) & Chest X-P & 1 day \\
RV perforation $(1810)$ & Not pacing at all & 2 days \\
A-lead dislodgement $(1810)$ & Not pacing at all & 9 months \\
A-lead dislodgement $(1810)$ & Undersensing during episode & 15 months \\
A-lead dislodgement $(1810)$ & Discovered at replacement & 4 months \\
A-lead dislodgement $(7271)$ & Noise signals & 1 month \\
A-lead fracture $(1820)$ & Noise signals & 9 months \\
V-lead fracture $(1810)$ & High pacing lead impedance & 9 months \\
V-lead fracture $(1810)$ & Inappropriate shocks & 15 months \\
V-lead fracture $(1810)$ & & 15 months \\
\hline
\end{tabular}

$D C$-ICD, dual-chamber implantable cardioverter defibrillator; SC-ICD, single-chamber implantable cardioverter defibrillator; $R V$, right ventricular; $V$, ventricular; $A$, atrial.

\section{Results}

The main objective of this study was to compare the incidence of major complications between DC-ICDs and SC-ICDs. Table 1 lists the clinical characteristics of the 2 groups of patients. The mean age was 66 years in the DCICD group and 62 years in the SC-ICD group (NS). The mean LVEF was lower in the DC-ICD group compared with the SC-ICD group $(29 \pm 12 \%$ vs $35 \pm 16 \%$, respectively; $\mathrm{p}=0.035)$. There was a statistically higher incidence of coronary artery disease in the SC-ICD group than in the
DC-ICD group (67\% vs 51\%, respectively; $\mathrm{p}<0.0076$ ), and the SC-ICD group had higher prevalence of aborted sudden cardiac death as the primary indication for implantation of the device ( $29 \%$ vs $19 \%$, respectively).

\section{Follow-up}

The average duration of follow-up was longer in the SCICD group than in the DC-ICD group (12 months and 9 months, respectively). During the follow-up period, there were 2 nonarrhythmic deaths, but neither of them was associated with ICD implantation. 

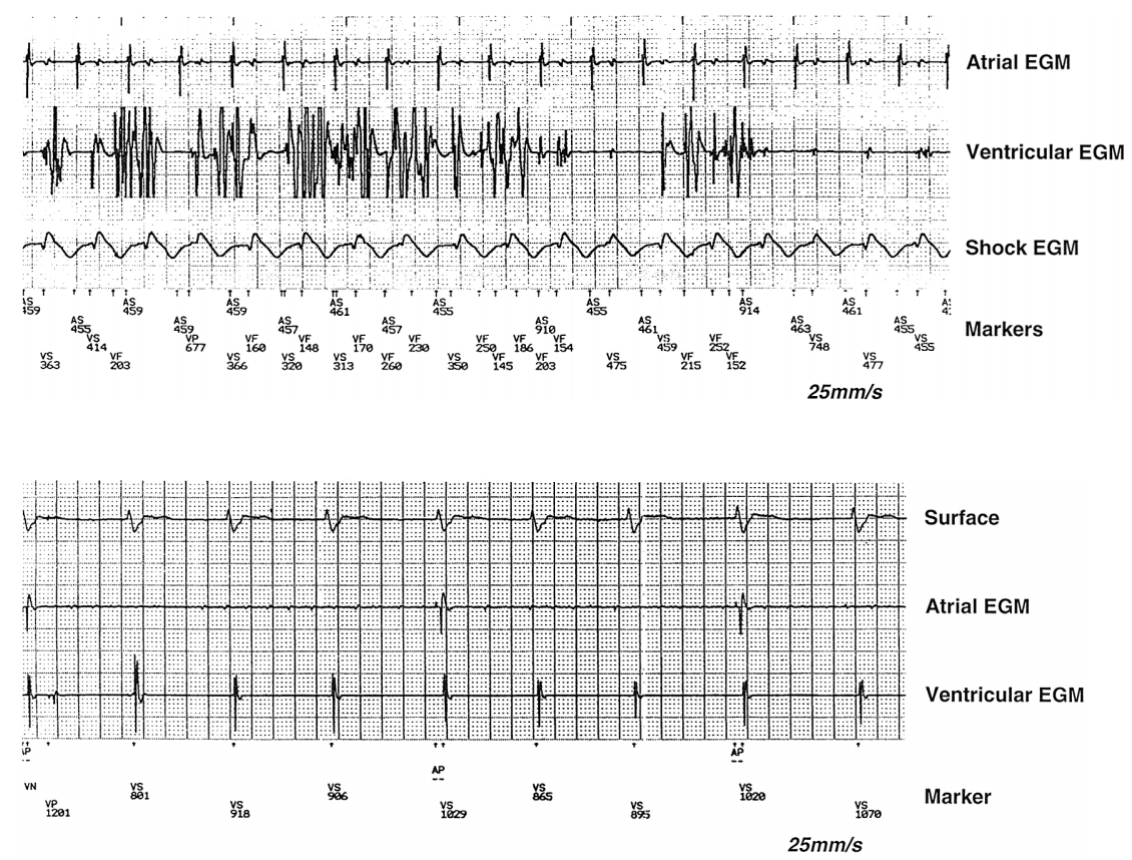

Fig 1. Noise on the ventricular pacing and sensing leads with high impedance is consistent with lead fracture. The patient received 7 shocks in 30 min 15 months after implantation. EGM, intracardiac electrogram.

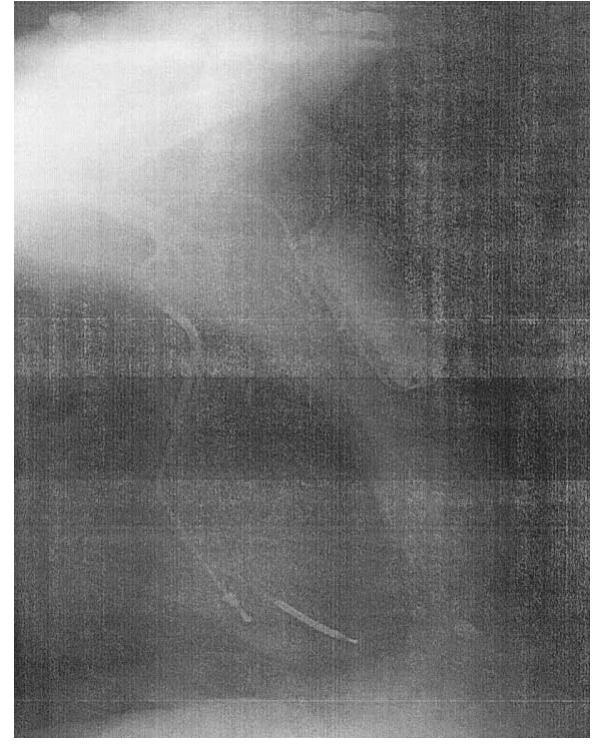

Fig 3. Fluoroscopic examination revealed dislodgement of the atrial lead to the right atrium. The patient was unable to pace through the atrial lead at the maximal current output of the device.

\section{Major Complications (Table 2)}

In the DC-ICD group, 16 major complications occurred in $12(16 \%)$ patients compared with 6 major complications in $6(6 \%)$ patients from the SC-ICD group. Lead-related complications occurred in 10 instances in $7(14 \%)$ patients in the DC-ICD group and in 2 instances in $2(2 \%)$ patients in the SC-ICD group, a significantly higher incidence with DC-ICD than SC-ICD.

Table 3 shows individual cases of lead complications with their type and the time after implantation that the event occurred. Early complications included cross-talk phenomenon and lead perforation. The day after implantation, 1 patient (SC-ICD) with a permanent pacemaker (VVI) because of sick sinus syndrome had the cross-talk phenom- enon between the previously placed right ventricular pacemaker lead and the ICD sensing lead, which inhibited pacing via the pacemaker. We replaced both leads. Another 4 patients (2 SC-ICD, 2 DC-ICD) required concomitant permanent pacemaker therapy, but no unfavorable results of sensing function were observed. One other patient (DCICD) received 7 shocks in $30 \mathrm{~min}$ at 15 months after the implant. We interrogated the device and found noise on the ventricular pacing and sensing lead with high impedance consistent with lead fracture (Fig 1). A new ventricular lead was placed on the high right ventricular septum, but the next day her chest X-ray showed a lead perforation. A subxiphoid window with a pericardial drain was placed when the lead was repositioned.

Lead dislodgment occurred in 5 patients (1 SC-ICD, 4 DC-ICD); the mean time of lead dislodgment from implantation was $6.1 \pm 5.9$ months. In the DC-ICD group, all were atrial-lead complications. One patient had spontaneous episodes of $\mathrm{P}$ wave undersensing 4 months after the implant, and his real time electrogram showed tiny $\mathrm{P}$ waves with a large ventricular electrogram recorded from the atrial lead. One patient who developed a lead infection 1 month after the implant had an atrial lead dislodgment discovered at the time of lead replacement. The other 2 patients were unable to pace through the atrial lead at the maximal current output of the device and a fluoroscopic examination revealed atrial lead dislodgement to the right atrium (Fig 2). One patient in the SC-ICD group who experienced inappropriate shocks had a malfunction of the device because of small $\mathrm{R}$ wave less than $1 \mathrm{mV}$, gross undersensing of the back-up pacing system and pacing failure for bradycardia (Fig 3).

Lead fractures ( 3 ventricular lead, 1 atrial lead) were diagnosed in 4 patients, all in the DC-ICD group, at 9-15 months after the implant (mean time for lead fracture, $12.0 \pm 3.5$ months). The diagnosis was made in 2 of them by noise signals, typically associated with maneuvers such as bending, stretching or sitting, and in 1 patient by high lead impedance on device interrogation. The other patient experienced inappropriate shocks. Each patient having lead 


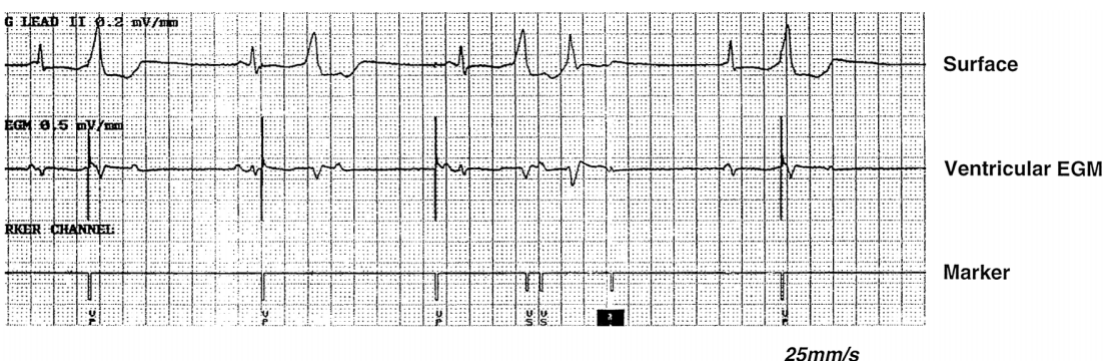

Fig 4. Small $\mathrm{R}$ wave less than $1 \mathrm{mV}$ and gross undersensing of the back-up brady system and pacing failure. The patient in the single-chamber ICD group experienced inappropriate shocks 15 months after implantation. EGM, intracardiac electrogram. fractures underwent a successful re-implantation operation. The site of lead fracture was localized by visual inspection to either the generator pocket $(n=1)$ or the cravicle rib $(n=2)$ or was undefined $(n=1)$.

Three patients in the DC-ICD group required explantation of the device and lead system because of lead infection. The infection was identified as Staphylococcus aureus (S. aureus) within 1 month of the implant in 2 patients, and the other patient developed severe pneumococcal infection 4 months after implant, which subsequently infected his ICD. After removal of the ICD system and a prolonged course of intravenous antibiotic therapy, we were able to successfully implant another non-thoracotomy defibrillation lead system in those 3 patients. To date, none of these has returned with a recurrent systemic infection.

\section{Discussion}

The major findings in our study were the high incidence of serious complications requiring surgical intervention in patients with DC-ICD compared with SC-ICD, and that most complications were related to the leads. Although rapid progress and improvement in design of ICDs have significantly contributed to decreasing the morbidity and mortality previously associated with ICD implantation, device-related complications are increasingly become a major hurdle to the long-term success of pectoral defibrillator implants with a transvenous defibrillator lead system. The reported incidence ranges from $2 \%$ to $37 \% \%^{4-6}$ and furthermore, the DC-ICD, which has 2 separate leads for sensing, appears to have more complications than a less sophisticated system. Of the complications noted in this study, lead dislodgment, lead fracture and infection warrant further investigation. As far as lead dislodgment is concerned, ventricular lead dislodgment is uncommon, with a rate of less than $2 \%$ in medical centers with expertise in ICD implantation? In the present study, only 1 ventricular lead was dislodged in the SC-ICD group $(1.0 \%$ rate of occurrence), compared with 4 instances of atrial lead dislodgement in the DC-ICD group. It may be more complicated to position an atrial lead in the small cephalic vein. The mean time for lead dislodgement was 6.1 \pm 5.9 months after implantation, which was earlier than lead fracture and consistent with a previous report. Moreover, all cases of dislodgement occurred during the first 6 months after initial DC-ICD implantation. Bardy et al described a marked reduction in the incidence of lead dislodgment in their more recent recipients, which they believed was the result of modifications in lead anchoring technique and operator experience!10

\section{Lead Fracture}

The rate of occurrence of lead fracture was significantly higher in the DC-ICD group. Schwartzman D et al reported that lead and patch fracture occurred later after implantation, and in the present cases lead fracture occurred 9-15 months after implantation in 5 patients in the DC-ICD group only. Although previous reports of the transvenous defibrillator lead systems described a low rate for lead fracture in 30 months of follow-up, it only developed approximately 40-50 months after implantation6 Therefore, the 12-month follow-up for the present study is rather short, and a longer follow-up is certainly warranted. Four of the 5 lead fractures occurred during the first 6 months after implantation and can be attributed to modifications in technique and operator experience.

\section{Infection}

The incidence of infection requiring explantation of the transvenous defibrillator system ranges from $2 \%$ to $5 \%, 1-13$ which is what we observed in the DC-ICD group only. The placement of a second lead is time-consuming and the longer operation time may have contributed to the trend toward more infections with the DC-ICD. Cannom et $\mathrm{al}^{4}$ also reported that early infection was S.aureus, and our results were consistent with that. One caution is our limited long-term follow-up, so further studies are warranted.

\section{Conclusion}

DC-ICDs using 2 separate leads for sensing were associated with a higher incidence of major complications than SC-ICDs and should be taken into consideration in the selection of device.

\section{Acknowledgments}

We gratefully acknowledge the assistance of many doctors and nurses who helped care for these patients in Good Samaritan Hospital, Los Angeles, CA, USA. We also thank Yong Park, RN for her help with the review of patients' data.

\section{References}

1. Brady GH, Hofer B, Johnson G, Kudenchuk PJ, Poole JE, Dolack GL, et al. Implantable transvenous cardioverter defibrillations. Circulation 1993; 87: $1152-1168$.

2. Saksea S, and the PCD Investigator Group. Clinical outcome of patients with malignant ventricular tachyarrhythmias and a multiprogrammable implantable cardioverter defibrillator implanted with or without thoracotomy: An international multicenter study. J Am Coll Cardiol 1994; 23: 1521 - 1530.

3. Zipes DP, Roberts D, and the Pacemaker Cardioverter-Defibrillator Investigators. Results of the International Study of the Implantable Pacemaker Cardioverter-Defibrillator: A comparison of epicardial and endocardial lead systems. Circulation 1995; 92: 59-65.

4. Schwartzman D, Nallamothu N, Callans DJ, Preminger MW, Gottlieb CD, Marchlinski FE. Postoperative lead related complications in patients with non-thoracotomy defibrillator lead systems. J Am Coll Cardiol 1995; 26: 776-786.

5. Lawton JS, Ellenbogen KA, Wood MA, Stambler BS, Herre JM, Nath 
S, et al. Implantable transvenous cardioverter-defibrillator leads to The dark side. Pacing Clin Electrophysiol 1996; 19: 1273-1278.

6. Mehta D, Nayak HM, Singson M, Chao S, Pe E, Camunas JL, et al. Late complications in patients with pectoral defibrillation lead systems: High incidence of insulation breakdown. Pacing Clin Electrophysiol 1998; 21: $1893-1900$.

7. Osswald S, Cron TA, Sterns L, Alt E, Stotts L, Coenen M. New ICD technologies: First clinical experience with dual chamber sensing for differentration of supraventricular tachyarrhythmias. Pacing Clin Electrophysiol 1998; 21: 292-295.

8. Le Franc P, Klug D, Lacroix D, Kouakam C, Jarwe M, Kacet S. Dualchamber implantable automatic defibrillators: Experiences apropos of 16 cases. Arch Mal Coeur Vaiss 1998; 91: 739-744.

9. Hayes DL. Pacemaker complications. In: Furman S, Hayes DL,
Holmes D, editors. Practice of cardiac pacing, 3rd edn. Armonk, NY: Futura; 1993: 537-570.

10. Jones GK, Dolack GL, Kudenchuk PJ. Complication following implantation of non-thoracotomy lead system cardioverter-defibrillations (abstract). J Am Coll Cardiol 1994; 23: 111A.

11. Kearney RA, Eisen HJ, Wolf JE. Non valvular infections of the cardiovascular system. Ann Intern Med 1994; 121: 219-230.

12. Kennergren C. Impart of implant techniques on complications with current implantable cardioverter-defibrillatior systems. Am J Cardiol 1996; 78(Suppl 5A): 15-20.

13. Higgins SL, Pak JP, Barone J, Williams SK, Bollinger FM, Whiting $\mathrm{SL}$, et al. The first year experience with the dual chamber ICD. Pacing Clin Electrophysiol 2000; 23: 18-25. 